\title{
Inertia Effects in Rheodynamic Lubrication of an Externally Pressurized Converging Thrust Bearing using Herschel-Bulkley Fluids
}

\author{
G. Alexander Raymand ${ }^{\dagger}$ and I. Jayakaran Amalraj \\ Department of Mathematics, SSN College of Engineering, Kalavakkam, Chennai, 603110, India \\ †Corresponding Author Email: alexanderraymand@gmail.com
}

(Received May 27, 2019; accepted December 17, 2019)

\begin{abstract}
Extreme industrial conditions require a bearing which can withstand high-speed operations, heavy load, high stiffness and so on. Therefore in this study, the combined effects of fluid inertia forces and non-Newtonian characteristic with Herschel-Bulkley fluid as lubricant in an externally pressurized converging thrust bearing have been contemplated. Avoiding complex calculation, the term inertia in the momentum equation is estimated by the mean value average method across the film thickness. A mathematical model for converged thrust bearing has been introduced. Using appropriate boundary conditions, thickness of the core, velocity profile, film pressure and the load carrying capacity of the bearing for various values of Herschel-Bulkley number $(N)$, Reynolds number $(R e)$, Power-law index $(n)$ and angle of convergence $(\varphi)$ have been numerically computed. Having worked with an externally pressurized flow through a narrow clearance between two convergent disks symmetrical with respect to $\mathrm{r}$ and $\mathrm{z}$ axis, it is found that the converged bearing performance such as pressure distribution and load carrying capacity increases notably. The results obtained in this study is found to be in agreement with the results of Jayakaran et al. (2012), for a particular case.
\end{abstract}

Keywords: Rheodynamic lubrication; Externally pressurized thrust bearing; Herschel-bulkley lubricants, Angle of convergence; Inertia effects.

\section{NOMENCLATURE}

$\begin{array}{ll}H-B & \text { Herschel-Bulkley } \\ h(r) & \text { varying film thickness } \\ h_{0} & \text { maximum film thickness } \\ h^{*}, p^{*}, r^{*}, z^{*} & \text { non-dimensional parameters of } \\ & h, p, r z \\ m & \text { stress growth exponent } \\ n & \text { power law index } \\ N & \text { Herschel-Bulkley number } \\ \eta_{1} & \text { consistency index } \\ \eta_{2} & \text { yield value } \\ p & \text { pressure of the film } \\ p_{a} & \text { atmospheric pressure } \\ Q & \text { flow rate } \\ R_{I} & \text { radius of film inlet }\end{array}$

$\begin{array}{ll}R_{2} & \text { radius of film outlet } \\ r, \theta, z & \text { cylindrical polar coordinates } \\ v_{c} & \text { velocity of the core region } \\ v_{r} & \text { velocity component in r direction } \\ v_{r} & \text { velocity component in z direction } \\ W & \text { load carrying capacity } \\ \delta(r) & \text { thickness of the yield surface } \\ \dot{\gamma} & \text { shear rate } \\ \tau & \text { deviatoric stress components } \\ \varphi & \text { angle of convergence } \\ \rho & \text { density of the fluid } \\ \delta^{*}, v_{c}^{*}, v_{r}^{*}, v_{z}^{*} & \text { non-dimensional parameters of } \\ & \delta(r), v_{c}, v_{r}, v_{z}\end{array}$

Generally, lubricants are classified into two types: Newtonian and non-Newtonian. At present, tribologists focus on non-Newtonian fluids that is characterized by yield value such as Bingham, Casson and Herschel Bulkley as its performance is high compared to Newtonian fluids. To optimize the performance of the bearing, the study of fluid inertia 
forces and a change in its design have been prioritized.

By principle, the considered bearing surfaces are separated by a fluid film which is created and maintained by external means. In the bearing considered for the study, the fluid film thickness varies according to angle of convergence $(\varphi)$; this likely leads to many advantages. Tribologists have keenly studied these advantages of rheodynamic lubrication in an externally pressurized thrust bearing by considering both Newtonian and nonNewtonian fluids.

In an externally pressurized thrust bearing, Amalraj et al. (2012) used Herschel-Bulkley fluid as lubricant to analyze the sinusoidal flow rate. Jurczak and Falicki (2016) investigated the externally pressurized bearing and squeeze film bearing with rough surface lubricated with non-Newtonian fluid analytically. Walicka et al. (2017a) analyzed the performance of externally pressurized curvulinear thrust bearing influenced by the rough surface and wall porosity lubricated by non-Newtonian fluid.

In rheodynamic lubrication, inertia forces are inertly developed as the fluid flows. This contributes to the performance of the bearing. Batra and Kandasamy (1989) analyzed the effects of inertia forces in the squeeze film bearing lubricated with viscoplastic fluid. Khalil et al. (1993) investigated the effects of convective and centrifugal inertia forces on the performance of an externally pressurized conical thrust bearings under a turbulent flow Usha and Vimala (2000) discussed inertia effects in a circular squeeze film bearing containing central air bubbles . The combined effects of fluid inertia and viscous forces have been investigated theoretically by Jayakaran et al. (2012) in an externally pressurized thrust bearing with circular geometry using Herschel-Bulkley fluid as lubricant. Walicka et al. (2017b) investigated the inertia and couple-stress effects on the pressure distribution and load-carrying capacity in a couple stress fluid flow with the clearance of a bearing formed by two coaxial surfaces of revolution. Shapour and Najafi (2017) analyzed the effect of inertial term of viscoplastic fluid flowing through a channel lined with higher compliant polymeric gel on hydroelastic stability of pressure driven flow. Udaya et al. (2011) theoretically investigated the effect of rotational inertia and pseudoplastic in an externally pressurized flow between parallel plates and concentric spherical surfaces. Alexander and Jayakaran (2019) theoretically investigated the inertial effects in Rheodynamic lubrication using Bingham fluid as lubricant.

H-B fluids are a class of non-Newtonian fluids that require a finite stress known as yield stress, which assists in the deform. Therefore, when the applied shear stress is below the yield stress value, these materials behave as a rigid body. Once the yield stress exceeds the material flow with a non-linear stress-strain relationship, it exhibits itself either as a shear thinning or shear thickening fluids. Many researchers like Alexandrou et al. (2001), Chan and Baird (2002), Huilgol et al. (2005), Vishwanath and
Kandasamy (2010) and Ponalagusamy and Priyadharshini (2019) have used H-B fluids to study their flow problems.

Tribologists have investigated inertial effect in externally pressurized thrust bearing with different types of fluids as lubricants, but only a few have analyzed the combined effects of fluid inertia forces and angle of convergence. Roy et al. (1993) analyzed the advantages of inertial effects for a converging film bearing over the uniform film bearing using visco-elastic fluid. He has observed that the converging bearing has more load carrying capacity and required less pump work for its functioning.

In this research work, a mathematical model Eq. (23) for novelly designed converged thrust bearing is introduced. Also, the combined effects of fluid inertia forces and angle of convergence on the performance of externally pressurized converging thrust bearing using Herschel-Bulkley fluid have been analyzed quantitatively. The core thickness, velocity, pressure and load carrying capacity for various values of Herschel-Bulkley number, Reynolds number and angle of convergence for visco-plastic fluid were computed numerically.

\section{MATHEMATICAL FORMULATION OF THE PROBLEM}

The investigation is presented in the upper half portion of the bearing by considering the symmetriness of the region between the circular plates of the bearing. The geometry of the converging bearing is as shown in Fig. (1)

The constitutive three-dimensional equation of a $\mathrm{H}$ B fluid is given by (Alexandrou et al. 2001)

$\tau=\left[\eta_{1}\left(\frac{D_{\mathrm{II}}}{2}\right)^{\frac{(n-1)}{2}}+\frac{\eta_{2}\left[1-\exp \left(-m \mid \sqrt{D_{\mathrm{II}} / 2}\right]\right]}{\sqrt{D_{\mathrm{II}} / 2}}\right] * \mathrm{D}$,

The second invariant of rate strain tensor $\mathrm{D}_{\text {II }}$ is given by $\mathrm{D}_{I I}=\mathrm{D}_{i j} \mathrm{D}_{i j}$, where $\mathrm{D}=\left[u+(u)^{T}\right]$. Further, for all practical purposes, one dimensional analog of Eq. 1 can be used, and it is given by Whorlow (1980)

$\tau=\eta_{2}+\eta_{1} \dot{\gamma}^{n}$, where $\dot{\gamma}$ represent shear rate

There is a region called core region where shear stress is less than the yield stress which moves with the constant velocity, $v_{c}$. Let the boundaries of the core be $z=-\frac{-\delta(r) h}{2}$ and $z=\frac{\delta(r) h}{2}$ as shown in Fig. (2).

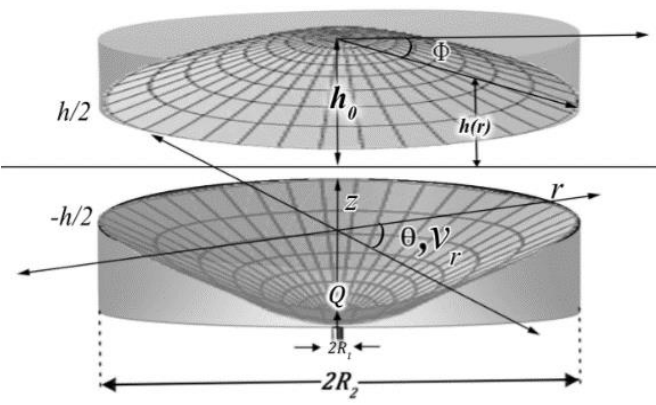

Fig. 1. Geometry of an Externally Pressurized Convergent Thrust Bearing. 


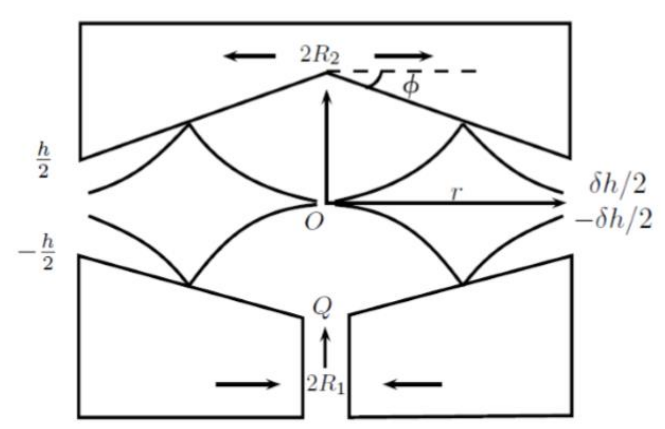

Fig. 2. Shape of the Core in an Externally Pressurized Thrust Bearing.

\subsection{Governing Equations \& Boundary Conditions}

Applying basic assumptions of the lubrication theory for thin films, the governing equation of HerschelBulkley fluid in an externally pressurized converging thrust bearing including inertia forces can be expressed as,

Equation of Continuity:

$\frac{1}{r} \frac{\partial}{\partial r}\left(r v_{r}\right)+\frac{\partial v_{z}}{\partial z}=0$

Equation of Momentum:

$\rho\left[v_{r} \frac{\partial v_{r}}{\partial r}+v_{z} \frac{\partial v_{r}}{\partial z}\right]+\frac{\partial p}{\partial r}=-\frac{\partial \tau_{r z}}{\partial z}$

$\frac{\partial p}{\partial z}=0$

$\tau_{r z}=\eta_{2}+\eta_{1}\left|\frac{\partial v_{r}}{\partial z}\right|^{n}$

The Eq.(3) - Eq.(6) are to be solved under the following boundary conditions

$v_{r}=0$ at $z= \pm \frac{h}{2}$

$v_{r}=v_{c}$, a constant, at $z= \pm \frac{\delta h}{2}$

$v_{r}$ is continuous, and $\frac{\partial v_{r}}{\partial z}=0$ at $\tau=\eta_{2}$

$$
p=p_{a} \text { at } r=R_{2}
$$

where $v_{c}$ is the core velocity and $p_{a}$ is atmospheric pressure.

\section{Solution OF THE PROBlem}

Now, by using the method of averaging inertia term in the Eq. (4), the following equation has been arrived at:

$\frac{\rho}{h}\left[\int_{-\frac{h}{2}}^{\frac{h}{2}}\left[v_{r} \frac{\partial v_{r}}{\partial r}+v_{z} \frac{\partial v_{r}}{\partial z}\right] d z\right]+\frac{d p}{d r}=-\frac{\partial \tau_{r z}}{\partial z}$

Using continuity Eq. (3) and boundary condition (6) and (7), the following equation has been arrived at:

$\frac{2 \rho}{h}\left[\frac{\partial}{\partial r} \int_{0}^{\frac{h}{2}} v_{r}^{2} d z+\frac{1}{r} \int_{0}^{\frac{h}{2}} v_{r}^{2} d z\right]+\frac{d p}{d r}=-\frac{\partial \tau_{r z}}{\partial z}$

Now, the modified pressure gradient has been introduced as $f \equiv \frac{2 \rho}{h}\left[\frac{\partial}{\partial r} \int_{0}^{\frac{h}{2}} v_{r}^{2} d z+\frac{1}{r} \int_{0}^{\frac{h}{2}} v_{r}^{2} d z\right]+\frac{d p}{d r}$

Equating (12) and (13), the following equation has been arrived at:

$f=-\frac{\partial \tau_{r z}}{\partial z}$

Integrating Eq. (14),

$\tau_{r z}=-f(z)+C$

By substituting $\tau_{\mathrm{r}}$ from Eq. (6) in to Eq. (15) and using the boundary conditions (7) and (8), the velocity distributions for the flow region is

$v_{r}=\left[\frac{n}{n+1}\right]\left[\frac{-f}{\eta_{1}}\right]^{\frac{1}{n}} * A$

Where, $A=\left[\left(z-\frac{\delta h}{2}\right)^{\frac{1}{n}+1}-\left(\frac{h}{2}-\frac{\delta h}{2}\right)^{\frac{1}{n}+1}\right]$

Where, $\frac{\delta h}{2} \leq z \leq \frac{h}{2}$

The velocity of the core region as

$v_{c}=-\left[\frac{n}{n+1}\right]\left[\frac{-f}{\eta_{1}}\right]^{\frac{1}{n}}\left[\frac{h}{2}-\frac{\delta h}{2}\right]^{\frac{1}{n}+1}$

Where, $0 \leq z \leq \frac{\delta h}{2}$

The equation of conservation of mass for externally pressurized bearing in an integral form is given by

$Q=4 \pi r \int_{0}^{\frac{\pi}{2}} v_{r} d z$

where $Q$ is the flow rate per unit width.

Using velocity distributions in (18) and integrating, we obtain

$Q=-\left[\frac{n(n \delta+n+1)}{(n+1)(2 n+1)}\right]\left[\frac{-f}{\eta}\right]^{\frac{1}{n}} * B$

Where, $B=\left[\frac{\left.\pi r h h^{\left[\frac{1}{n}+2\right.}\right]}{2^{\left[\frac{1}{n}\right]}}\right]$

Considering the equilibrium of an element in the

yield surface $-\frac{\delta h}{2} \leq z \leq \frac{\delta h}{2}$, it is found that

$f=\frac{2 \eta_{2}}{\delta(r) h}$

Hence, Eq. (19) becomes

$f=\frac{2 \eta_{1} Q^{n}\left[\frac{(n+1)(2 n+1)}{n}\right]^{n}}{\pi^{n} r^{n} h^{(2 n+1)}(1-\delta)^{(n+1)}}$

Elimination $f$ from (20) and (21), the algebraic equation for determining the thickness of the yield surface $\delta(r)$ can be obtained as

$$
\begin{aligned}
& \frac{\delta^{\frac{1}{n}}}{(1-\delta)^{\left(\frac{1}{n}+1\right)}(n \delta+}n+1) \\
&=\left[\frac{n}{(n+1)(2 n+1)}\right] * C
\end{aligned}
$$

Where, $C=\left[\frac{\pi r h^{2}}{Q}\left(\frac{\eta_{2}}{\eta_{1}}\right)^{\frac{1}{n}}\right]$

The variation of film thickness of the lubricant in the converging bearing can be defined as 
$h(r)=h_{0}-h_{0}\left(\frac{r}{R_{2}}\right) \tan \phi$

where, $h(r)$ represents the varying film thickness between the plates, $\mathrm{h} 0$ is the maximum film thickness at the center of the bearing, and $\varphi$ is the angle of convergence.The following non-dimensional parameters are introduced.

$r^{*}=\frac{r}{R_{2}} ; \delta^{*}=\delta\left(r^{*}\right) ; p^{*}=\frac{p}{\left(\frac{Q^{n} \eta_{1}}{\pi^{n} h_{0}^{2 n+1} R_{2}^{n-1}}\right)}$

$h^{*}=\frac{h}{h_{0}} ; z^{*}=\frac{z}{h} ; N=\frac{\pi R_{2} h_{0}^{2}}{Q}\left(\frac{\eta_{2}}{\eta_{1}}\right)^{\frac{1}{n}}$

Using the non-dimensional quantities in Eq.(16),(17) we get the velocity of the fluid in the flow region as

$$
v_{r}^{*}=\frac{3}{2}\left[\frac{\left(1-\delta^{*}\right)^{2}-\left(2 z^{*}-\delta^{*}\right)^{2}}{r^{*}\left(1-r^{*} \tan \phi\right)\left(2+\delta^{*}\right)\left(1-\delta^{*}\right)^{2}}\right] ;
$$

Where $\frac{\delta^{*} h^{*}}{2} \leq z^{*} \leq \frac{h^{*}}{2}$

Also, the velocity of the core region is

$v_{c}^{*}=\frac{3}{2}\left[\frac{1}{r^{*}\left(1-r^{*} \tan \phi\right)\left(2+\delta^{*}\right)}\right]$

Where $0 \leq z^{*} \leq \frac{\delta^{*} h^{*}}{2}$

Substituting $v_{r}, v_{c}$ and $f$ from Eqs.(16),(17) and (21) in Eq. (13) non-dimensionalized pressure gradient has been obtain as

$\frac{d p^{*}}{d r^{*}}=\frac{2\left[\frac{(n+1)(2 n+1)}{n}\right]^{n}}{r^{* n}\left(1-r^{*} \tan \phi\right)^{2 n+1}(1-\delta)^{n+1}(\delta+n+1)^{2}}-\{\mathrm{E}\}$

where,

$\mathrm{E}=R e *\left[\frac{\frac{(2 n+1)}{(3 n+2)}}{4 r^{* 2}\left(1-r^{*} \tan \phi\right)^{2}(n \delta+n+1)^{2}}\right] *\left[K_{1}-K_{2}\right]$

where,

$K_{1}=\left[\delta^{*}(4 n+3)+2(n+1)^{2}\right]\left(\frac{-1}{r^{*}}+\frac{\tan \phi}{1-r^{*} \tan \phi}\right)$

$K_{2}=\frac{\partial \delta^{*}}{\partial r^{*}}\left(\frac{4 n^{3} \delta^{*}+3 n^{2} \delta^{*}+n^{2}+n}{n \delta^{*}+n+1}\right)$

A non-dimensinalized non linear algebraic equation has been obtained to determining the core thickness from Eq.(22)

$\frac{\delta^{*} \frac{1}{n}}{\left(1-\delta^{*}\right)^{\frac{1}{n}+1}\left(n \delta^{*}+n+1\right)}=\left[\frac{n * N\left(1-r^{*} \tan \phi\right)^{2} r^{*}}{(n+1)(2 n+1)}\right]$

The roots of the the Eq.(28) determine the shape of the plug core region. By differentiation Eq.(28) w.r.t $r^{*}$ it is obtained that

$\frac{d \delta^{*}}{d r^{*}}=$

$\frac{(n+1)(2 n+1)}{N * n}\left(\frac{-1+3 r^{*} \tan \phi}{r^{* 2}\left(1-r^{*} \tan \phi\right)^{3}}\right)$

$\left[\frac{\delta^{*}}{1-\delta^{*}}\right]^{\frac{1}{n}}\left(\frac{n \delta^{*}}{-2 n^{2} \delta^{* 2}-2 n \delta^{*}-n-1}\right)$

The pressure distribution can be obtained by substituting (28) in (26) and integrating using boundary condition $(8)$,

$P^{*}-P_{a}^{*}=\int_{r^{*}}^{1}\left[\frac{d p^{*}}{d r^{*}}\right] d r^{*}$ where, $R e=\frac{\rho Q^{2-n} h_{0}^{2 n-1}}{\eta_{1} \pi^{2-n} R_{2}^{n-3}}$ is Reynolds number. The pressure distribution has been calculated for different values of Herschel-Bulkley number, Reynolds number and Angle of convergence numerically. Again, the load carrying capacity $W$ for the externally pressurized thrust bearing can be obtained by integrating the pressure over the entire region. By this

$W=\int_{R^{*}}^{1}\left[P^{*}-P_{a}^{*}\right] r^{*} d r^{*}$

where, $R^{*}=\frac{R_{1}}{R_{2}}$ is the ratio of inside to outside radius of the bearing. This integration is performed numerically for various values of the $\operatorname{Re}, B$, and $\varphi$.

\section{RESULTS AND DISCUSSION}

The behavior of the core $\left(h^{*}\right)$ for various values of power-law index $(n)$, Herschel-Bulkley number $(N)$, and angle of convergence $(\varphi)$ at every point of radius $\left(r^{*}\right)$ is computed numerically and the results are shown in Figs. (3)-(6).

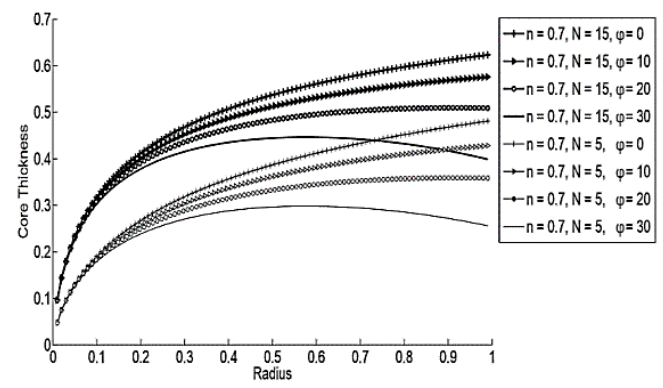

Fig. 3. Core thickness for variation along the radius for $n=0.7$

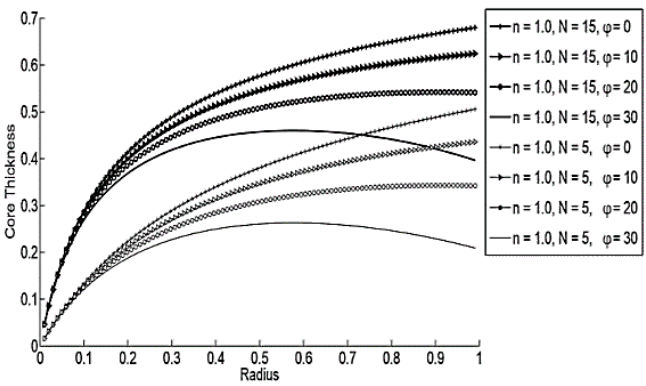

Fig. 4. Core thickness for variation along the radius for $n=1$.

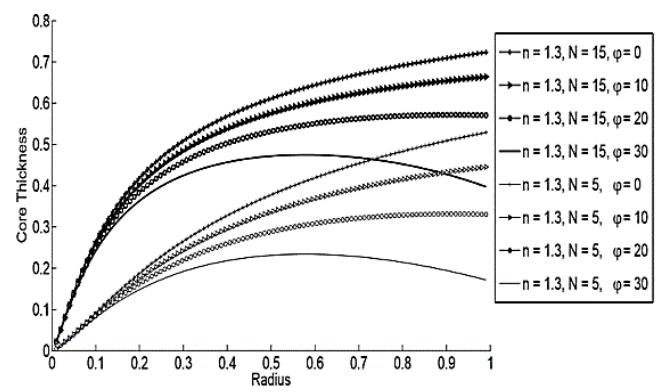

Fig. 5. Core thickness for variation along the radius for $n=1.3$. 
Figures (3), (4) and (5) depicts core thickness for power-law index $n=0.7,1,1.3$ along the radial direction. It increases gradually from the center and decreases as it approaches the periphery as the angle of convergence increases for a given $\mathrm{H}-\mathrm{B}$ number $(N$ $=5 \& 15$ ). In Fig. (6), when $\varphi=30$ variation in Core thickness is marginal between power-law index $n=$ $0.7,1,1.3$ for particular $\mathrm{H}-\mathrm{B}$ number $(N=15)$. The formation of unyielding core occurs symmetrically in the middle of the region between the circular disc of the bearing. Moreover, the core thickness increases as the power-law index increases for a particular Herschel-Bulkley number.

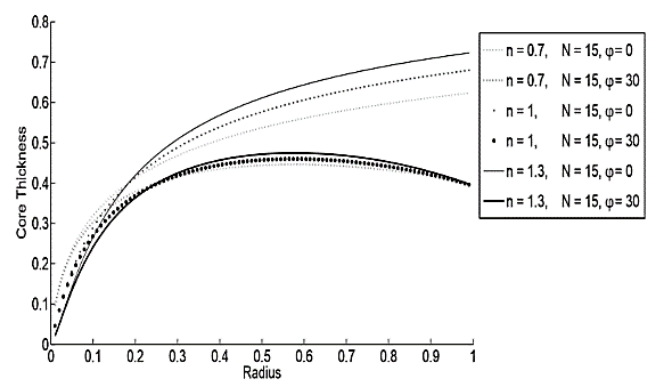

Fig. 6. Core thickness variation for particular Herschel-Bulkley Number $N=15$.

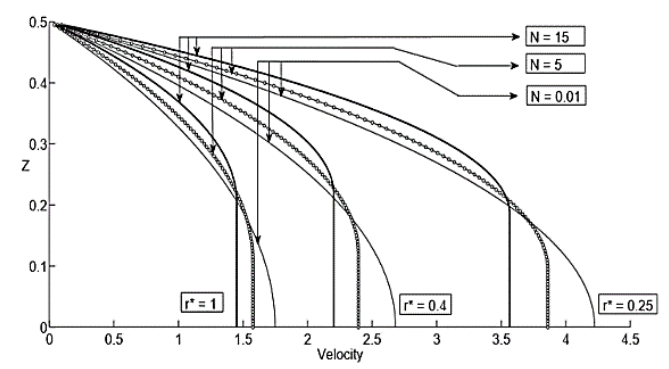

Fig. 7. Velocity profile for particular power-law index $n=1$.

The velocity profile for various angle of convergence, H-B number \& power law-index $(\varphi, N$ $\& n$ ) along the axial direction( $\mathrm{z}^{*}$ ) for various values of the radius $\left(\mathrm{r}^{*}\right)$ are depicted in the Fig. (7). The thickness of the core as observed earlier, is reflected in the velocity profile. The velocity profile becomes parabolic if we consider the symmetric region between the plates as H-B number $\lim _{\mathrm{N} \rightarrow 0}$, which represents Newtonian fluid. The distribution of the film pressure in the radial direction has been obtained for various values of H-B number $(N=5)$, Reynolds number $(R e=0,0.1,0.2)$, power-law index $(n=0.7,1,1.3)$ and angle of convergence $(\varphi=0,20)$ these are shown in Figs. (8)-(11). There is a considerable increase of inertial effect in pressure distribution as power-law index increases. This is depicted in Figs. (8)- (10) respectively, moreover, the pressure is maximum at the orifice and gradually decreases as it moves towards the periphery of the bearing along the radial direction.

It is observed that an increase in pressure is more significant when angle of convergence $(\varphi)$ is increased for specific power-law index $(n)$. However, the quantum of increase is marginal for high Reynolds number. The inertial effect on pressure distribution near the orifice of the bearing is found to be appreciable and this is shown in Fig. (11) for a particular angle of convergence $(\varphi=5)$.

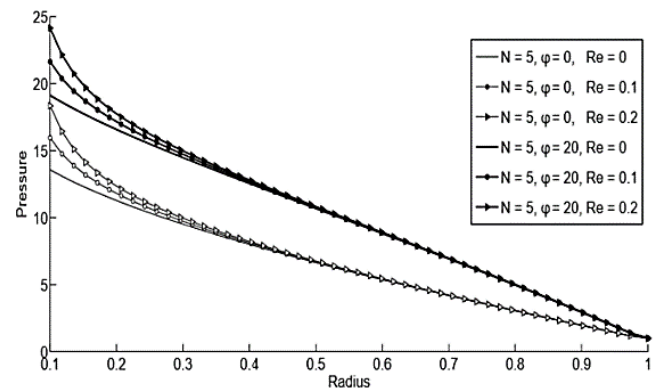

Fig. 8. Inertia effect in Pressure distribution for particular Power-law index $n=0.7$.

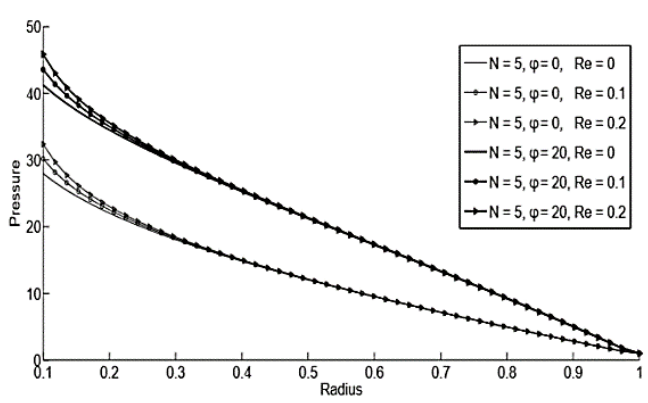

Fig. 9. Inertia effect in Pressure distribution for particular Power-law index $n=1$.

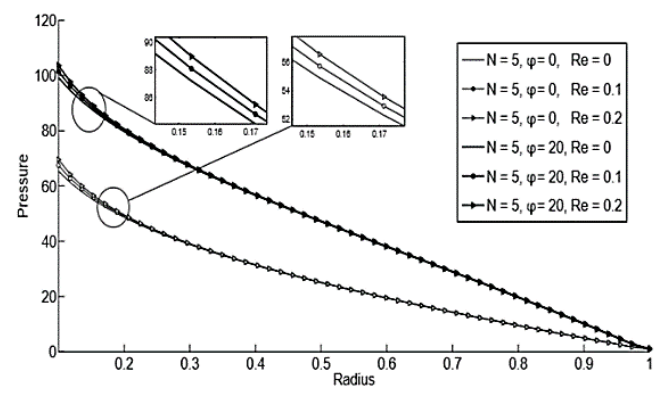

Fig. 10. Inertia effect in Pressure distribution for particular Power-law index $n=1.3$.

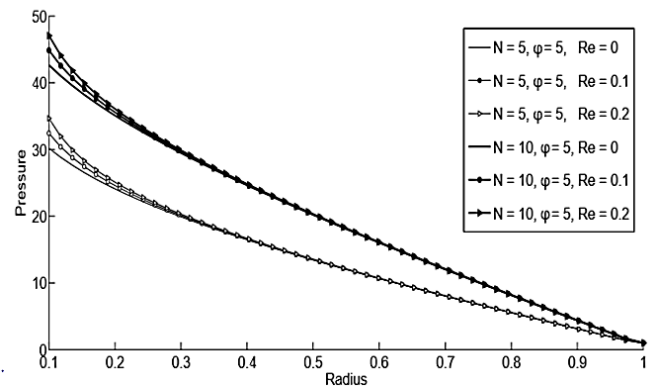

Fig. 11. Inertia effect in Pressure distribution for particular Angle of Convergence $\varphi=5$.

The numerically computed results of load carrying capacity for particular Herschel-Bulkley number $N=$ $5 \&$ specific power-law index value $n=0.7, n=1$ \& $n=1.3$ are given in the table (1)-(3) for various 
Reynolds number $(R e) \&$ angle of convergence $(\varphi)$. The load carrying capacity of the bearing has been found to increase significantly as the power-law index $(n)$ of the fluid increases for a fixed $H-B$ number.

Also, there is a significant increase in load carrying capacity as the angle of convergence increases for a particular power-law index $(n)$. Further, as the Reynolds number increases the increase in load carrying capacity is marginal for the particular power-law index $(n)$, angle of convergence and H-B number.

Table 1 Load Capacity for different value of $R e$ $\& \varphi$ for $n=0.7$

\begin{tabular}{|c|c|c|c|c|c|}
\hline \multirow{2}{*}{$\mathrm{N}=5$} & \multicolumn{5}{|c|}{ Reynolds number $\left(\times 10^{-1}\right)$} \\
\hline & 0 & 1 & 2 & 3 & 4 \\
\hline$\varphi=0$ & 2.40 & 2.54 & 2.69 & 2.83 & 2.99 \\
\hline$\varphi=5$ & 2.60 & 2.75 & 2.90 & 3.05 & 3.20 \\
\hline$\varphi=10$ & 2.87 & 3.02 & 3.17 & 3.33 & 3.48 \\
\hline$\varphi=15$ & 3.23 & 3.38 & 3.54 & 3.70 & 3.84 \\
\hline
\end{tabular}

Table 2. Load Capacity for different value of $R e$ $\& \varphi$ for $n=1$

\begin{tabular}{|c|c|c|c|c|c|}
\hline \multirow{2}{*}{$\mathrm{N}=5$} & \multicolumn{5}{|c|}{ Reynolds number $\left(\times 10^{-1}\right)$} \\
\hline & 0 & 1 & 2 & 3 & 4 \\
\hline$\varphi=0$ & 4.25 & 4.39 & 4.53 & 4.67 & 4.81 \\
\hline$\varphi=5$ & 4.72 & 4.86 & 5.00 & 5.14 & 5.28 \\
\hline$\varphi=10$ & 5.32 & 5.46 & 5.60 & 5.74 & 5.89 \\
\hline$\varphi=15$ & 6.13 & 6.27 & 6.42 & 6.56 & 6.70 \\
\hline
\end{tabular}

Table 3 Load Capacity for different value of $R e$ $\& \varphi$ for $n=1.3$

\begin{tabular}{|c|c|c|c|c|c|}
\hline $\mathrm{N}=5$ & \multicolumn{5}{|c|}{ Reynolds number $\left(\times 10^{-1}\right)$} \\
\hline & 0 & 1 & 2 & 3 & 4 \\
\hline$\varphi=0$ & 8.8 & 8.9 & 9.1 & 9.2 & 9.3 \\
\hline$\varphi=5$ & 9.9 & 10 & 10.1 & 10.3 & 10.4 \\
\hline$\varphi=10$ & 11.3 & 11.4 & 11.6 & 11.7 & 11.8 \\
\hline$\varphi=15$ & 13.2 & 13.4 & 13.5 & 13.7 & 13.8 \\
\hline
\end{tabular}

Table 4 Load Capacity for different value of $R e$ $\& N$ for $n=0.7$

\begin{tabular}{|c|c|c|c|c|c|}
\hline \multirow{2}{*}{$\mathrm{N}=5$} & \multicolumn{5}{|c|}{ Reynolds number $\left(\times 10^{-1}\right)$} \\
\hline & 0 & 1 & 2 & 3 & 4 \\
\hline$\varphi=5$ & 2.60 & 2.75 & 2.90 & 3.05 & 3.20 \\
\hline$\varphi=10$ & 3.25 & 3.40 & 3.55 & 3.70 & 3.85 \\
\hline$\varphi=15$ & 3.78 & 3.94 & 4.09 & 4.24 & 4.39 \\
\hline
\end{tabular}

Table 5 Load Capacity for different value of $R e$ $\& N$ for $n=1$

\begin{tabular}{|c|c|c|c|c|c|}
\hline \multirow{2}{*}{$\mathrm{N}=5$} & \multicolumn{5}{|c|}{ Reynolds number $\left(\times 10^{-1}\right)$} \\
\hline & 0 & 1 & 2 & 3 & 4 \\
\hline$\varphi=5$ & 4.72 & 4.86 & 5.00 & 5.14 & 5.28 \\
\hline$\varphi=10$ & 6.95 & 7.09 & 6.81 & 7.37 & 7.51 \\
\hline$\varphi=15$ & 9.09 & 9.23 & 9.37 & 9.51 & 9.65 \\
\hline
\end{tabular}

Table 6 Load Capacity for different value of $R e$ $\& N$ for $n=1.3$

\begin{tabular}{|c|c|c|c|c|c|}
\hline \multirow{2}{*}{$\mathrm{N}=5$} & \multicolumn{5}{|c|}{ Reynolds number $\left(\times 10^{-1}\right)$} \\
\hline & 0 & 1 & 2 & 3 & 4 \\
\hline$\varphi=5$ & 9.9 & 10.0 & 10.2 & 10.3 & 10.4 \\
\hline$\varphi=10$ & 17.7 & 17.8 & 17.1 & 18.1 & 18.2 \\
\hline$\varphi=15$ & 26.3 & 26.4 & 26.5 & 26.7 & 26.8 \\
\hline
\end{tabular}

Table 7 Change in Percentage in load Capacity with respect to $R e=0$ for different values of $\varphi$ and $n=0.7$

\begin{tabular}{|c|c|c|c|c|c|}
\hline \multirow{2}{*}{$\mathrm{N}=5$} & \multicolumn{5}{|c|}{ Reynolds number $\left(\times 10^{-1}\right)$} \\
\hline & 0 & 1 & 2 & 3 & 4 \\
\hline$\varphi=0$ & 6.21 & 12.42 & 18.62 & 24.83 & 31.04 \\
\hline$\varphi=5$ & 5.76 & 11.52 & 17.28 & 23.03 & 28.79 \\
\hline$\varphi=10$ & 5.27 & 10.55 & 15.82 & 21.09 & 26.36 \\
\hline
\end{tabular}

Table 8 Change in Percentage in load Capacity with respect to $R e=0$ for different values of $\varphi$ and $n=1$

\begin{tabular}{|c|c|c|c|c|c|}
\hline $\mathrm{N}=5$ & \multicolumn{5}{|c|}{ Reynolds number $\left(\times 10^{-1}\right)$} \\
\hline & 0 & 1 & 2 & 3 & 4 \\
\hline$\varphi=0$ & 3.26 & 6.52 & 9.79 & 13.05 & 16.31 \\
\hline$\varphi=5$ & 2.97 & 5.94 & 8.91 & 11.89 & 14.86 \\
\hline$\varphi=10$ & 2.66 & 5.33 & 7.99 & 10.66 & 13.32 \\
\hline
\end{tabular}

Table 9 Change in Percentage in load Capacity with respect to $R e=0$ for different values of $\varphi$ and $n=1.3$

\begin{tabular}{|c|c|c|c|c|c|}
\hline \multirow{2}{*}{$\mathrm{N}=5$} & \multicolumn{5}{|c|}{ Reynolds number $\left(\times 10^{-1}\right)$} \\
\hline & 0 & 1 & 2 & 3 & 4 \\
\hline$\varphi=0$ & 1.52 & 3.04 & 4.56 & 6.08 & 7.60 \\
\hline$\varphi=5$ & 1.37 & 2.74 & 4.10 & 5.47 & 6.84 \\
\hline$\varphi=10$ & 1.21 & 2.42 & 3.63 & 4.84 & 6.05 \\
\hline
\end{tabular}

The results of load carrying capacity for a particular angle of convergence $(\varphi=5)$, different HerschelBulkley numbers, Reynolds number \& specific power-law index values $n=0.7, n=1 \& n=1.3$ are given in the table (4)-(6). It is observed from the analysis that there is an appreciable increase in the value of the load capacity as H-B number increases. This is due to the fact that Herschel-Bulkley fluids, being a thick viscous model, show a high load carrying capacity. However, the inherent inertial effect is marginal.

The percentage of increase in load carrying capacity for different values of Reynolds number $(R e)$, Angle of convergence $(\varphi)$, power-law index $(n=0.7, n=1 \&$ $n=1.3$ ) and Herschel-Bulkley number $(N=5)$ are given in the tables (7)-(9). It has been observed that due to inertia effect the percentage of increase in load carrying capacity is significant for low Angle of convergence. 
Tables (10)-(12) shows that due to inertia effect the percentage of increase in load carrying capacity is significant for low Herschel-Bulkley number.

Table 10 Change in Percentage in load Capacity with respect to $R e=0$ for different values of $\varphi$ and $\boldsymbol{n}=0.7$

\begin{tabular}{|c|c|c|c|c|c|}
\hline $\mathrm{N}=5$ & \multicolumn{5}{|c|}{ Reynolds number $\left(\times 10^{-1}\right)$} \\
\hline & 0 & 1 & 2 & 3 & 4 \\
\hline$\varphi=5$ & 5.76 & 11.52 & 17.28 & 23.03 & 28.79 \\
\hline$\varphi=10$ & 4.64 & 9.29 & 13.93 & 18.57 & 23.21 \\
\hline$\varphi=15$ & 4.00 & 8.00 & 12.00 & 15.99 & 19.99 \\
\hline
\end{tabular}

Table 11 Change in Percentage in load Capacity with respect to $R e=0$ for different values of $\varphi$ and $n=1$

\begin{tabular}{|c|c|c|c|c|c|}
\hline $\mathrm{N}=5$ & \multicolumn{5}{|c|}{ Reynolds number $\left(\times 10^{-1}\right)$} \\
\hline & 0 & 1 & 2 & 3 & 4 \\
\hline$\varphi=5$ & 2.97 & 5.94 & 8.91 & 11.89 & 14.86 \\
\hline$\varphi=10$ & 2.00 & 4.01 & 6.01 & 8.02 & 10.02 \\
\hline$\varphi=15$ & 1.52 & 3.05 & 4.57 & 6.10 & 7.62 \\
\hline
\end{tabular}

Table 12 Change in Percentage in load Capacity with respect to $R e=0$ for different values of $\varphi$ and $n=1.3$

\begin{tabular}{|c|c|c|c|c|c|}
\hline \multirow{2}{*}{$\mathrm{N}=5$} & \multicolumn{5}{|c|}{ Reynolds number $\left(\times 10^{-1}\right)$} \\
\hline & 0 & 1 & 2 & 3 & 4 \\
\hline$\varphi=5$ & 1.37 & 2.74 & 4.10 & 5.47 & 6.84 \\
\hline$\varphi=10$ & 0.75 & 1.51 & 2.26 & 3.01 & 3.77 \\
\hline$\varphi=15$ & 0.50 & 1.00 & 1.50 & 2.00 & 2.50 \\
\hline
\end{tabular}

Moreover, the percentage of increase in the load carrying capacity increases significantly as the Reynolds number increases. The inertia plays a small role in the bearing performance. For a particular angle of convergence $(\varphi=0)$ which corresponds to a flat externally pressurized thrust bearing, these results on pressure distribution and load carrying capacity of the bearing are found to be in agreement with the results of (Jayakaran et al. 2012) for a particular case.

\section{CONCLUSION}

The above investigation shows:

1. Using Herschel-Bulkley fluid as lubricant in the bearing increases the pressure and load carrying capacity.

2. Angle of convergence in bearing design also significantly enhances the bearing performance. However, the effect of fluid inertia on the bearing performance is found to be marginal.

\section{ACKNOWLEDGMENTS}

The authors thank the Principal and management of SSN institutions for their support in carrying out this work.

\section{REFERENCES}

Alexandrou, A. N., T. M. McGilvreay and G. Burgos (2001). Steady Herschel-Bulkley fluid flow in three-dimensional expansions. Journal of NonNewtonian Fluid Mechanics 100, 77-96.

Alexander Raymand G. and Amalraj I. J. (2019). Inertia Effects in Rheodynamic Lu-brication of an Externally Pressurized Converging Thrust Bearing using Bingham Fluids. Journal of Applied Fluid Mechanics 12(2), 587-594.

Amalraj I. J. S. Narasimman and A. Kandasamy (2012). Rheodynamic lubrication of an externally pressurized thrust bearing using Herschel-Bulkley fluid with sinusoidal injection. Journal of Applied Fluid Mechanics 5(4), 71-79.

Amalraj I. J. (2019). Inertia Effects in Rheodynamic Lubrication of an Externally Pressurized Converging Thrust Bearing using Bingham Fluids. Journal of Applied Fluid Mechanics 12(2), 587-594.)

Batra, R. L. and A. Kandasamy (1989). Inertia effects in rheodynamic lubrication of a squeeze film bearing. Wear, 273-282.

Huilgol. R. R. and Z. You (2005). Application of the augmented lagrangian method to steady ppe flows of Bingham, Casson and HerschelBulkley fluids. Journal. of Non-Newtonian Fluid Mechanics 128, 126-143.

Jayakaran Amalraj, I., S. Narasimman and A. Kandasamy (2012). Inertia Effects in an Externally Pressurized Thrust Bearing Using Herschel - Bulkley Lubricants. American Journal of Computational and Applied Mathematics 2(3), 79-85.

Jurczak, P., and J. Falicki, (2016). Pressure Distribution in a Squeeze Film Spherical Bearing with Rough Surfaces Lubricated by an Ellis Fluid. International Journal of Applied Mechanics and Engineering 21(3), 593-610.

Khalil, M. F., S. Z. Kassab and A. S. Ismail (1993). Effect of inertia forces on the performance of externally pressurized conical thrust bearing under turbulent flow condition. Wear, 166, 15516.

Ponalagusamy, R., and S. Priyadharshini (2019). A numerical model on pulsatile flow of magnetic nanoparticles as drug carrier suspended in Herschel-Bulkley fluid through an arterial stenosis under external magnetic field and body force. International Journal of Computer Mathematics 96 (9), 1763-1786.

Roy, S. J., S. Padhy, L. K. and Bhopa (1993). Inertia effect in externally pressurized thrust bearing with converging and diverging film using viscoelastic lubricant. Acta Mechanica 96, 1-12.

Chan, T. W. and D. G. Baird (2002). An evaluation of a squeeze flow rheometer for the rheological characterization of a filled polymer with a yield 
G. Alexander Raymand and I. Jayakaran Amalraj / JAFM, Vol. 13, No. 4, pp. 1245-1252, 2020.

stress. Rheol Acta 41, 245-256.

Udaya, P. S., R. S. Gupta and V. K. Kapur (2011). Effects of Inertia In The Steady State Pressurised Flow Of A Non-Newtonian Fluid Between Two Curvilinear Surfaces of Revolution: Rabinowitsch Fluid Model. Chemical and Process Engineering, 32(4), 333349.

Usha, R. and P. Vimala (2000). Inertia effects in circular squeeze film containing a central air bubble. Fluid Dynamics Research 26, 149-155.

Vishwanath K. P. and A. Kandasamy (2010). Inertia effects in circular squeeze film bearing using Herschel-Bulkley lubricants. Applied Mathematical Modelling 34, 219-227.
Walicka, A., E. Walicki, P. Jurczak and J. Falicki (2017a). Influence of Wall Porosity and Surfaces Roughness on the Steady Performance of an Externally Pressurized Hydrostatic Conical Bearing Lubricated by Rabinowitsch Fluid. Journal of Applied Mechanics and Engineering 22(3), 717-737.

Walicka, A., P. Jurczak and J. Falicki (2017b). Inertia and Couple-Stress Effects in a Curvilinear Thrust Hydrostatic Bearing. J. of Applied Mechanics and Engineering 22(2), 759-767.

Whorlow, R. W. (1980). Rheological techniques, Halstead Press. 